\title{
Phenotypic performance of progeny from UK sires selected on female fertility beef EBVs
}

K L Moore, R Mrode, B Lowman, T Roughsedge

SAC, Penicuik, United Kingdom

Email: kirsty.moore@sac.ac.uk

Introduction Growth and carcass trait EBVs have been produced for the UK beef industry since the 1990's, with maternal 200 day milk being the only EBV available for female fertility (FF). To provide the UK beef industry with direct and maternal EBVs for FF traits a research project was undertaken to consider trait definitions, model development and genetic parameter estimation (Roughsedge et al., 2005a). Limousin heritability estimates from the study were generally low, ranging from 0.04 (calving interval (CI)) to 0.27 (age at first calving (AFC)). Being sex-limited traits means that sires have to wait until their daughters reproduce until performance information is available for a sire. Consequently, the sire is often approximately 5 years of age by the time daughter performance records for FF traits are available for use in genetic evaluation. For carcass traits, the sires own performance information is known at approximately 1-2 years of age. The generally low heritabilities and performance records of daughters only available late in life means that sires are generally older when they receive accurate FF EBVs. Despite these challenges, significant genetic improvement for FF traits is achievable (Roughsedge et al., 2005b). In 2007 the EGENES/Signet genetic evaluations incorporated for the first time EBVs for AFC, CI, calving ease (direct and maternal components), life span and gestation length (direct and maternal components) (Coffey et al. 2007). However, feedback from industry suggests that these FF EBVs have been under utilised in the UK beef industry. The aim of this work was to demonstrate the value of the FF EBVs for breeding replacement cows.

Materials and methods A 2009 routine Limousin genetic evaluation was used to select sire subsets representative of the distribution tails for each trait. To ensure sufficient data only sires with relatively high accuracies were considered. There were almost 25,000 sires recorded in total, with 10,220 and 4,278 sires having accuracies greater than 50\%, respectively for AFC and CI. From these sires and for each trait, the 200 best and worst ranked sires were selected. To evaluate selected sire subsets, phenotypic records from contemporary groups (CG) containing progeny of selected sires were considered. The CG definitions were the same as in the national evaluation and were based on the birth herd and date of birth for AFC and calving herd, date of first calving and a six month season for CI. The individual phenotypic records were deviated from the CG mean. These deviations from the mean were then compared for the different sire subset's progeny. These deviations were only within the CGs and were not adjusted for other effects (i.e. age effects) in the model. A 'historic' evaluation was undertaken using current models and parameters but with data truncated from 1999. This run was then used to compare the EBVs of high accuracy sires with when they were younger and less accurate.

Results Table 1 provides EBV summaries of selected sires and progeny phenotypic deviations from CG means. For example, the CI EBV spanned 43 days for high accuracy sires, with 23.2 days being the average difference between sires identified as being the best and worst for CI. A daughter from a best sire had a CI on average 7 days shorter than her CG, while progeny of a worst sire had a calving interval on average 9 days later than her CG. Similar trends were observed for all the FF traits (results not shown). Between 50 and 71 of selected sires were aged 0-5 years in the 1999 run. These sires were ranked the same at both time points but with greater variation (because EBVs less accurate) when they were young bulls compared to when they were high accuracy older sires. The best sires had AFC and CI EBVs of -0.08 (0.08) and -2.1 (5.0), respectively as young bulls and the worst sires had AFC and CI EBVs of $0.11(0.10)$ and 4.6 (4.0), respectively as young bulls.

Table 1 Summary of the female fertility EBVs for subsets of high accuracy sires representative of the best and worst and their progenies mean phenotypic deviations (PMPD) from contemporary group means

\begin{tabular}{lcccccc}
\hline \hline & \multicolumn{3}{c}{ Selected 200 best sires } & \multicolumn{3}{c}{ Selected 200 worst sires } \\
\cline { 2 - 7 } Trait $*$ & EBV Mean (Range) & N progeny & PMPD (std) & EBV Mean (Range) & N progeny & PMPD (std) \\
\hline AFC & $-0.16(-0.33$ to -0.13$)$ & 1973 & $-21(101)$ & $0.23(0.19$ to 0.33) & 2349 & $17(110)$ \\
CI & $-9.8(-19.5$ to -7.0$)$ & 2938 & $-7(41)$ & $13.4(10.9$ to 23.5) & 3260 & $9(48)$ \\
\hline \hline
\end{tabular}

* $\mathrm{AFC}=$ Age at first calf (score based on days (phenotype in days)), $\mathrm{CI}=$ Calving interval (days)

Conclusions This work shows that progeny phenotypes from high accuracy sires are representative of FF EBVs from the UK beef evaluations. Selection using the UK FF EBVs allows breeders to breed for cows that calve earlier and easier, have shorter calving intervals, increased longevity and improved milking abilities. However, this work has highlighted an important challenge for the UK beef industry to consider. The lower heritability and late in life performance measures of the FF traits mean that sires are older (5+years) before accurate EBVs are available. Therefore selection strategies for FF traits need to be a compromise between reduced generation intervals (i.e. selecting younger bulls) and increased EBV accuracy (i.e. selecting older more accurate bulls).

Acknowledgements Thank you to QMS for funding this project and Signet performance recording for the use of the data.

\section{References}

Coffey, M., Krzyzelewski, T., Roughsedge, T. and Mrode, R. 2007. Interbull Bulletin 36, 1-5

Roughsedge, T., Amer, P.R., Thompson, R. and Simm, G. 2005a. Journal of Animal Science 83, 2319-2329

Roughsedge, T., Amer, P.R., Thompson, R. and Simm, G. 2005b. Animal Science 81, 221-232 